\title{
Effect of temperature and strain rate on the compressive behaviour of supramolecular polyurethane
}

\author{
Xuegang Tang ${ }^{1, a}$, Clive R. Siviour ${ }^{1}$, C. Paul Buckley ${ }^{1}$, Antonio Feula ${ }^{2}$, and Wayne Hayes ${ }^{2}$ \\ ${ }^{1}$ Department of Engineering Science, Oxford University, Parks Road, Oxford OX1 3PJ, UK \\ ${ }^{2}$ Department of Chemistry, University of Reading, Whiteknights, Reading RG6 6AD, UK
}

\begin{abstract}
Supramolecular polyurethanes (SPUs) possess thermoresponsive and thermoreversible properties, and those characteristics are highly desirable in both bulk commodity and value-added applications such as adhesives, shape-memory materials, healable coatings and lightweight, impact-resistant structures (e.g. protection for mobile electronics). A better understanding of the mechanical properties, especially the rate and temperature sensitivity, of these materials are required to assess their suitability for different applications. In this paper, a newly developed SPU with tuneable thermal properties was studied, and the response of this SPU to compressive loading over strain rates from $10^{-3}$ to $10^{4} \mathrm{~s}^{-1}$ was presented. Furthermore, the effect of temperature on the mechanical response was also demonstrated. The sample was tested using an Instron mechanical testing machine for quasi-static loading, a home-made hydraulic system for moderate rates and a traditional split Hopkinson pressure bars (SHPBs) for high strain rates. Results showed that the compression stress-strain behaviour was affected significantly by the thermoresponsive nature of SPU, but that, as expected for polymeric materials, the general trends of the temperature and the rate dependence mirror each other. However, this behaviour is more complicated than observed for many other polymeric materials, as a result of the richer range of transitions that influence the behaviour over the range of temperatures and strain rates tested.
\end{abstract}

\section{Introduction}

The mechanical behaviour of polymeric materials has long been a topic of keen interest for scientists across many disciplines, and it is well-known that strain rate and temperature have significant influence on the mechanical properties [1,2]. Depending on their (processing) behaviour at elevated temperatures, normally, polymers are divided into three main classes: thermoplastics, thermosets and rubbers. Among them, rubbers, or alternatively elastomers, are soft, flexible and elastic materials at room temperature and are applied in a wide range of applications, such as tires, tubes, seals and gloves. Crosslinking is necessary to obtain the typical rubber properties, such as elasticity, high toughness and solvent resistance, since this severely restricts the motion of the chain molecules. Currently, the main industrial techniques for rubber cross-linking are sulfur vulcanization and peroxide curing, which results in a threedimensional network via sulfur bridges in the first case and $\mathrm{C}-\mathrm{C}$ bonds in the second. However, the formation of an irreversible network upon cross-linking prevents melt (re)processing of both thermosets and elastomers, and this gives them a distinct processing disadvantage compared to thermoplastics [3].

Supramolecular polymers, a newly-developing group of materials, can be loosely defined as those in which the monomers are held together by noncovalent interactions [4]. Therefore, a reversible network can

\footnotetext{
${ }^{\text {a }}$ Corresponding author: xuegang.tang@eng.ox .ac.uk
}

be formed, which is sensitive to temperature, and can be destroyed and reconstructed easily during processing and post-processing. This provides those materials with unique thermoresponsive and thermoreversible properties, thereby delivering dramatic viscosity changes over well-defined and tunable temperature ranges: at room temperature they behave like elastomers, but at elevated temperature are low viscosity liquids. These characteristics offer huge advantages in processing and also provide unique properties, which are highly desirable in both bulk commodity and valued-added applications. By understanding the mechanical properties of these materials and the microscopic mechanisms controlling their mechanical behaviour, we will be better able to design supramolecular polymers and supramolecular polymerbased systems for specialized application. However, the mechanical properties, especially at high strain rate have been relatively uncharacterized to date.

In this study, a newly-developed supramolecular polyurethane (SPU) through hydrogen bond and pi-pi stacking was fabricated, and the effects of temperature and strain rate on the behaviour under compression were investigated. SPUs are sensitive to temperature, and the response at elevated temperatures is governed by the dissociation of non-covalent band such as hydrogen bonds and $\pi-\pi$ stacking, as well as the time temperature superposition principle for traditional polymers [5]. These effects were considered when describing the rate and temperature dependence of the material, which was not expected to follow the usual form associated with 'traditional' polymeric materials.

This is an Open Access article distributed under the terms of the Creative Commons Attribution License 4.0, which permits unrestricted use, distribution, and reproduction in any medium, provided the original work is properly cited. 


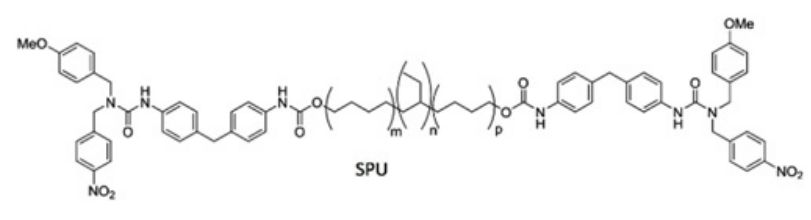

Figure 1. The chemical structure of SPU.

\section{Experimental procedure}

\subsection{Chemical structure of SPU}

Previous research suggests that hydrogen bond and $\pi-\pi$ stacking can be introduced to polymer to form noncovalent interaction among polymer chains [6-9], and those non-covalent interactions present significant temperature sensitivity, which shows rich transitions at narrow temperature and frequency range. Therefore, those materials offer a unique chance to investigate the transitions of polymers, especially the effect of those transitions on stress-strain behaviour, at narrow temperature and/or frequency regime compared with traditional polymers such as polycarbonate and poly(methyl metacrylate) etc., which normally need large temperature and frequency range to observe those transitions [10-12].

In this study, a supramolecular polyurethane (SPU) was synthesized by an established approach using Krasol HLBH-P2000 and the diisocyanate MDI with the control of end group to achieve $\pi-\pi$ stacking and hydrogen bond, at the University of Reading [8], and the specific chemical structure is shown in Fig. 1. Both Krasol HLBH-P2000 and the diisocyanate prepolymer are thick viscous liquids at room temperature whereas in contrast fabricated SPU is a solid material indicating the crucial role of the formation of supramolecular networks. Detail procedures of the synthesis and structure characterization will be reported in a separate paper.

\subsection{Sample preparation}

The fabricated SPU was moulded as cylindrical compression samples at the temperature of $50^{\circ} \mathrm{C}$ for half hour with the diameter of $5 \mathrm{~mm}$ and thickness of about $2.5 \mathrm{~mm}$. The size of the test samples was based on previous work on compression testing, especially at high strain rate. This suggested that length-to-diameter ratios of 1:2 or less are desirable in the testing of low-impedance materials on high rate test systems, in order to provide adequate strain gage signals and improve the time taken to reach static stress equilibrium. Also, a length-to-diameter ratio of 1:2 has been shown to be optimal in negating the effects of radial and longitudinal inertia in the specimen $[10,13,14]$.

\subsection{Experimental set-up}

The samples were tested across a wide range of strain rates from $10^{-3}$ to $10^{4} \mathrm{~s}^{-1}$, at room temperature (RT, $19.0 \pm$ $0.5^{\circ} \mathrm{C}$ ) and a temperature range from $-30^{\circ} \mathrm{C}$ to $30^{\circ} \mathrm{C}$ at the strain rate of $10^{-2} \mathrm{~s}^{-1}$. An Instron (model 5982) was used for quasi-static loading at the strain rates from $10^{-3} \mathrm{~s}^{-1}$ to $10^{-1} \mathrm{~s}^{-1}$. An environmental chamber with liquid nitrogen attachment was used during tests for temperature control. The specimens were loaded using hardened steel anvils. Standard Instron load cells were used to measure the forces applied to the specimen, whilst a clip extensometer was used to measure the displacement of the anvils, from which specimen strain was calculated, in addition a designed feedback loop between the extensometer and the actuator was used to ensure constant true strain rate control over the duration of the tests.

Medium rate experiments were performed using an in-house hydraulic system built at Oxford University to access strain rates between 1 and $50 \mathrm{~s}^{-1}$. A strain gauge based load cell with a high natural frequency has been designed, and is against a rigid steel plate. A Linear Variable Differential Transformer (LVDT) was used to measure the displacement of the sample, from which strain can be calculated.

High strain rate experiments were performed using a split-Hopkinson bar system (SHPB). The SHPB consists of a $1000 \mathrm{~mm}$ long input bar and a $500 \mathrm{~mm}$ long output bar. The striker bar is $500 \mathrm{~mm}$ long, and all bars are $12.7 \mathrm{~mm}$ diameter. Both the input and output bars are instrumented with foil strain gauges, the input bar halfway along its length, and the transmitted bar $50 \mathrm{~mm}$ from the bar specimen interface. Magnesium alloy bars were used in this work for the test. This low stiffness, high strength material gives large gauge signals even when testing soft polymers, but are strong enough not to yield at the impact speeds required for the higher strain rate experiments. The tests and calculation was based on reference handbooks $[15,16]$.

All specimens were lubricated with a thin layer of paraffin wax on both faces to avoid barrelling effect.

Parallel plate oscillatory shear was performed with an Anton Paar Physica MCR 301 rheometer. For the temperature sweep at a single frequency of $5 \mathrm{~Hz}$ with the deformation of $0.1 \%$ to guarantee it's in the linear viscoelastic region, the samples were placed on the rheometer with well contact between parallel plates. The sample were then subjected to a cooling temperature ramp of $2{ }^{\circ} \mathrm{C} / \mathrm{min}$ to $-30^{\circ} \mathrm{C}$, and held at this temperature for 5 minutes. After this step they were heated up to $60{ }^{\circ} \mathrm{C}$ at $2{ }^{\circ} \mathrm{C} / \mathrm{min}$. For the isothermal frequency sweeps, the samples were again placed in the rheometer and maintained at the selected temperature for 5 minutes, and then the frequency sweeps were performed between 0.1 and $100 \mathrm{rad} / \mathrm{s}$ at a strain amplitude of $0.1 \%$. Three cycles of tests were measured, and the results show excellent reproducibility. During all of these steps the dynamic shear moduli (G', G') of the samples were recorded.

\section{Results and discussion}

\subsection{Temperature dependence}

Figure 2 shows the variation of complex shear modulus. It can be seen that the SPU presents the transition zone to flow, plateau zone (rubbery), and the transition zone to glassy behaviour from 60 to $-30^{\circ} \mathrm{C}$, which are: lower than $-20^{\circ} \mathrm{C}$, from -20 to $10^{\circ} \mathrm{C}$, and higher than $10^{\circ} \mathrm{C}$ respectively. Especially, when the temperature is higher than $30^{\circ} \mathrm{C}$, a rapid drop of storage modulus can be observed owing to the dissociation of the supramolecular 


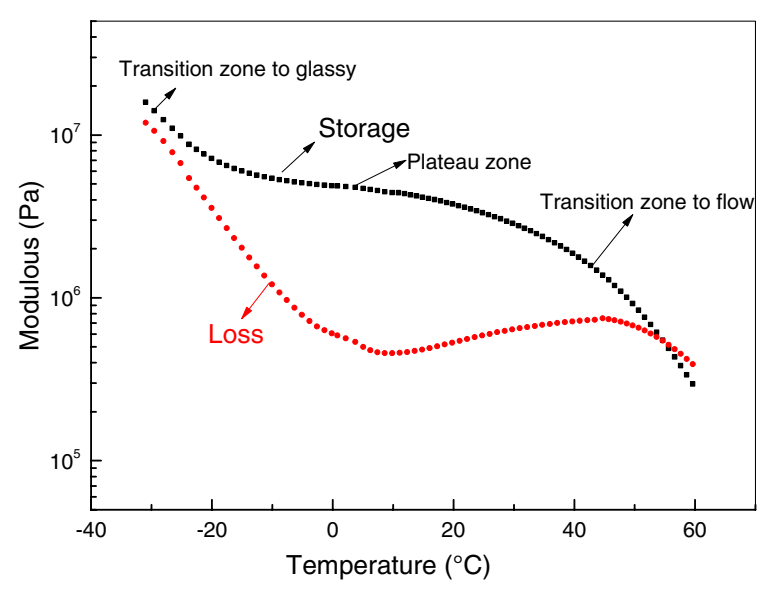

Figure 2. Variation of complex shear modulus with temperature at the frequency of $5 \mathrm{~Hz}$.

polyurethane network, resulting in a large degree of softening. This shows that for SPU, rich transitions occur in a narrow temperature range, and may indicate different stress-strain behaviour with those transitions.

The compressive stress-strain curves of SPU at the strain rate of $10^{-2} \mathrm{~s}^{-1}$ with different temperature are shown in Fig. 3a. Generally, the compressive stressstrain response of SPU is similar to other elastomers, but it can also be observed that temperature affects the stress-strain behaviour of the SPU significantly. For example, at a temperature of $30^{\circ} \mathrm{C}$, after an initial deformation, the stress-strain curve is flat; there is a large flat region in the stress-strain curve following. However, with decreasing temperature, strain hardening at large deformations become more and more obvious. The compressive stress-strain behaviour can be divided into three categories: 1) a general flat regime at large deformation after initial deformation (about 25\% strain) for the temperature from 30 to $10^{\circ} \mathrm{C}$; 2) almost linear behaviour during the whole deformation from 0 to $-20^{\circ} \mathrm{C}$; and 3 ) a significant increase in stress at large deformations when the temperature is below $-30^{\circ} \mathrm{C}$. In order to parameterise these curves for comparison to other data sets, the stress at $25 \%$ strain is plotted against temperature, Fig. 3b. These data show an almost linear increase of strength as the temperature drops from 30 to $0{ }^{\circ} \mathrm{C}$, then a plateau down to $-20^{\circ} \mathrm{C}$, with strength again increasing as the temperature drops further.

The observed behaviour agrees well with the rheometer test results, corresponding to the three distinguish physical status of SPU: transition zone to flow, plateau zone (rubbery), and transition zone to glassy behaviour at different temperature. Overall, the data strongly demonstrate the temperature sensitivity of the SPU.

\subsection{Rate dependence}

Polymers, due to their viscoelastic nature, present behaviour with both temperature and time (frequency) dependence during deformation. Time/temperature superposition, based on the equivalency between time (or frequency) and temperature, usually is applied to polymers, which suggests that the viscoelastic variations occur relatively quick at higher temperatures can be made
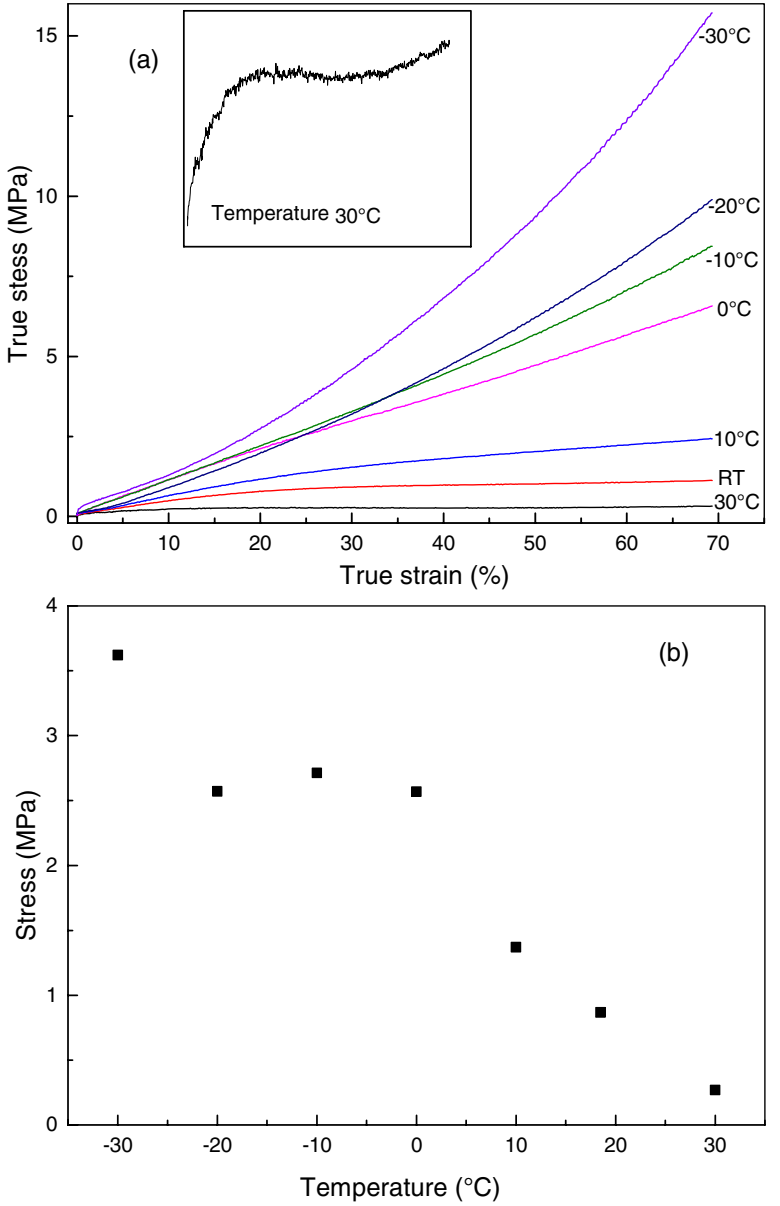

Figure 3. (a) Compressive stress-strain response of SPU and (b) stress (at 25\% strain) at the strain rate of $10^{-2} \mathrm{~s}^{-1}$ with different temperature.

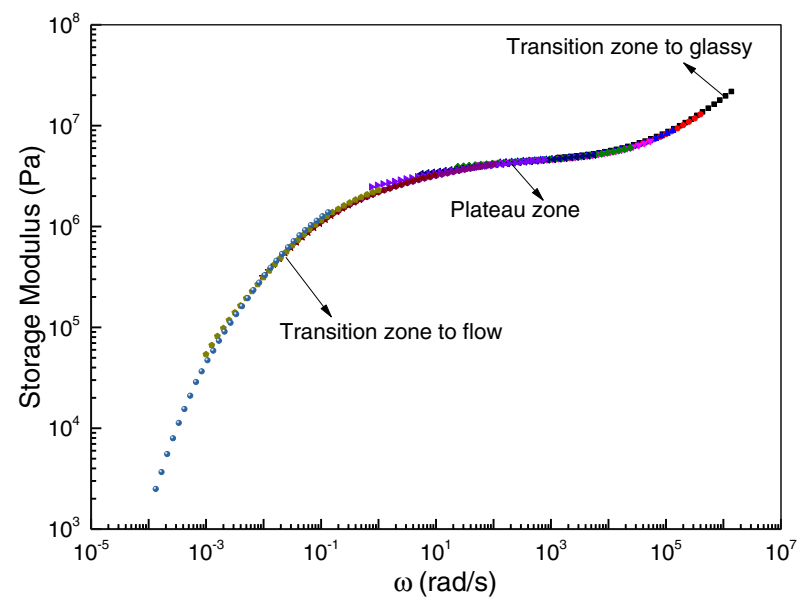

Figure 4. Variation of complex shear modulus with temperature frequency (master curve at the reference temperature of $20^{\circ} \mathrm{C}$ ). Colours indicate different temperatures.

to appear as if they occur at longer times or lower frequencies simply by shifting the data with respect to time (or frequency) [5]. In this work, frequency sweeps from temperatures -30 to $50{ }^{\circ} \mathrm{C}$ were used to generate a master curve exploiting the time/temperature superposition principle to predict the frequency dependence of SPU over a large frequency range, far beyond the reach of 

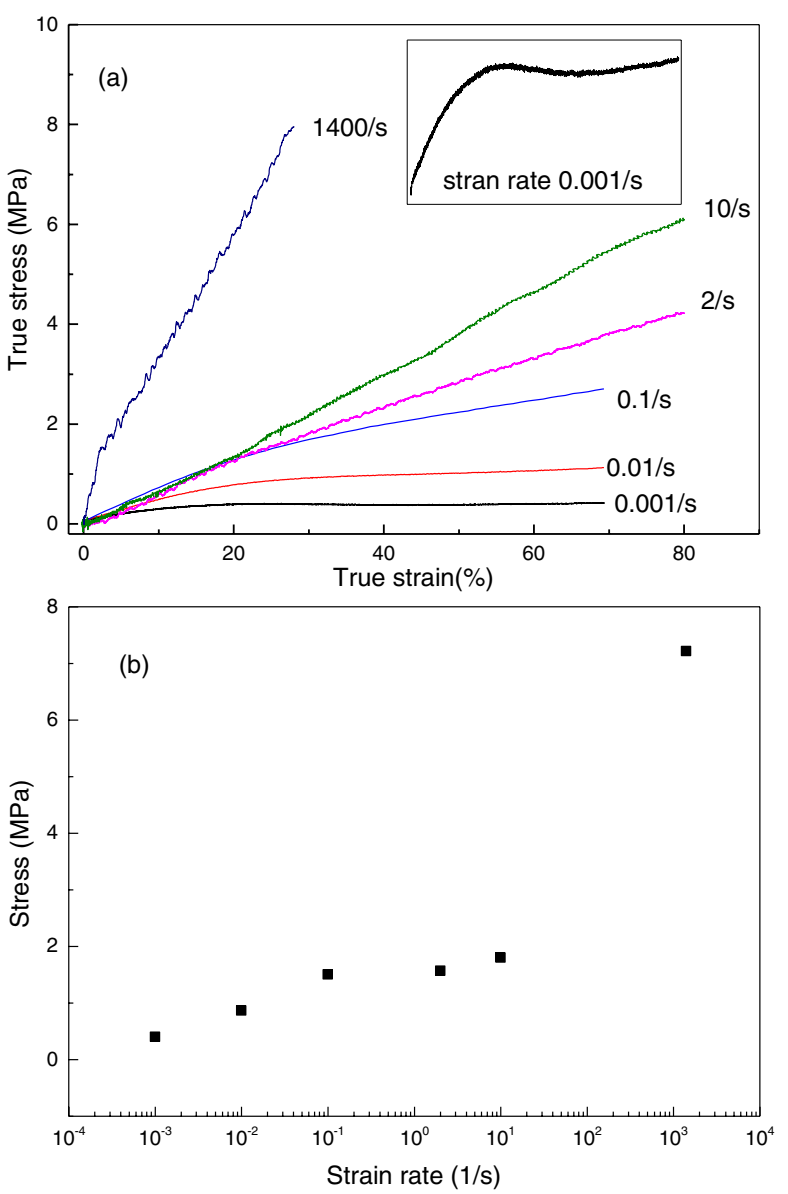

Figure 5. (a) Compressive stress-strain response of SPU and (b) stress (at 25\% strain) at room temperature with different strain rate.

the test machine. Results show that time/temperature superposition works well for SPU, indicated by the satisfactory overlapping achieved during the shifting of the curves. The corresponding master curve is shown in Fig. 4. Similar to the temperature dependence, the master curve of frequency also shows three distinguish status with the variation of frequency: transition zone to flow, plateau zone (rubbery), and the transition zone to glassy behaviour at a narrow frequency range.

Figure 5a provides a comparison of representative high-rate true stress-true strain curve with representative low to moderate rate curves, which suggests significant changes of the deformation behaviour, indicating the high sensitivity of the SPU to strain rate. For example, at the lowest strain rate $\left(10^{-3} \mathrm{~s}^{-1}\right)$, the stress-strain behaviour of SPU is similar to typical thermoplastic polymer: showing an initial elastic region, followed by a nonlinear elastic region and yield (at a strain of about 25\%), then strain softening followed by strain hardening [17]. However, as the strain rate increases, even still at low strain regime $\left(10^{-2}\right.$ and $\left.10^{-1} \mathrm{~s}^{-1}\right)$, yielding and strain softening disappear, and the behaviour is similar to traditional thermoplastic polyurethanes [18]. At moderate rate, the curves present linear behaviour during the whole deformation.

Figure $5 \mathrm{~b}$ shows SPU stress (at the strain of $25 \%$, which corresponds the yield point of SPU at the lowest strain rate) as a function of strain rate across all of the tested strain rates. Within the low rate regime, the stress is found to increase linearly with the logarithm of strain rate, indicating that the behaviour in this regime could be accurately explained and/or predicted in terms of a single activated process, which is in agreement with some previous works on polymers [10]. However, it is interesting to observe that there is a plateau regime at moderate rate, and followed by a sharp jump at high strain rates. Therefore, a bilinear behaviour as a function of strain rate is not enough to describe the rate dependence of the SPU. This is maybe due to the changes of response of SPU on strain rate: at low rate, it behaviours more like thermoplastic, and with the rising of strain rate, it behaviours more like rubber, and finally a transition to glassy. To some extent, these observations are entirely consistent with the observed temperature dependence, both of which exhibit an increase in the supported stress, then a plateau then a further increase. However, it is noted that the observed stresses and the shapes of the stress strain curves at the lowest rates are not the same as those at the highest temperatures (e.g. no strain softening at the highest temperature of $30^{\circ} \mathrm{C}$ ), and this will need further investigation.

\section{Conclusions}

A supramolecular polyurethane (SPU), which forms networks through hydrogen bonding and pi-pi stacking has been experimentally studied in compression at varying strain rates and temperatures, and in oscillatory shear rheometry. Time/temperature superposition was employed to construct the master curve for SPU to predict the properties at large frequency range. Dynamic rheometer results indicate that SPU presents a transition zone to flow, plateau zone (rubbery), and transition zone to glassy behaviour from 60 to $-30^{\circ} \mathrm{C}$. Compression tests show that the SPU presents significant temperature and rate dependence, which is in agreement with the transitions observed in the rheometry. The results also suggest that a plateau regime has to be incorporated into the bilinear behaviour model to describe the rate dependence for SPU from low strain rate to high strain rate. However, additional testing and analysis is required to confirm these trends.

The authors would like to thank EPSRC for support (EP/J010715/1 and EP/J011436/1), in addition to the University of Reading for access to instrumentation in the Chemical Analysis Facility and Cray Valley for the supply of Krasol HLBH-P2000.

\section{References}

[1] E.M. Arruda, M.C. Boyce, R. Jayachandran, Mech. Mater. 19, 193 (1995)

[2] L.C.A. van Breemen, T.A.P. Engels, E.T.J. Klompen, D.J.A. Senden, L.E. Govaert, J. Polym. Sci. Part B Polym. Phys. 50, 1757 (2012)

[3] V.D. Mee, MAJ (Mark), Technische Universiteit Eindhoven (2007) 
[4] A.W. Bosman, R.P. Sijbesma, E.W. Meijer, Mater. Today 7, 34 (2004)

[5] J.D.V. Ferry, Viscoelastic properties of polymers (Wiley, New York, 1980)

[6] S. Burattini, B.W. Greenland, D.H. Merino, W. Weng, J. Seppala, H.M. Colquhoun, W. Hayes, M.E. Mackay, I.W. Hamley, S.J. Rowan, J. Am. Chem. Soc. 132, 12051 (2010)

[7] S. Burattini, H.M. Colquhoun, J.D. Fox, D. Friedmann, B.W. Greenland, P.J.F. Harris, W. Hayes, M.E. Mackay, S.J. Rowan, Chem. Commun. 44, 6717 (2009)

[8] P.J. Woodward, D.H. Merino, B.W. Greenland, I.W. Hamley, Z. Light, A.T. Slark, W. Hayes, Macromolecules 43, 2512 (2010)

[9] P. Cordier, F. Tournilhac, C. Soulie-Ziakovic, L. Leibler, Nature 451, 977 (2008)

[10] A.D. Mulliken, M.C. Boyce, M, Int. J. Solids Struct. 43, 1331 (2006)
[11] C.R. Siviour, S.M. Walley, W.G. Proud, J.E. Field, Polymer 46, 12546 (2005)

[12] A.D. Mulliken, in: Massachusetts Institute of Technology. Dept. of Mechanical Engineering (Massachusetts Institute of Technology, 2006)

[13] N.N. Dioh, P.S. Leevers, J.G. Williams, Polymer 34, 4230 (1993)

[14] C.M. Roland, Rubber Chem. Technol. 79, 429 (2006)

[15] G.T. Gray III, in ASM Handbook. Vol. 8, ASM International: Materials Park, Ohio, 2000, pp. 462476

[16] G.T. Gray III, W.R. Blumenthal, in ASM Handbook. Vol.8, ASM International: Materials Park, Ohio, 2000, pp. 488-496

[17] J.L. Jordan, C.R. Siviour, B.T. Woodworth, Shock Compression of Condensed Matter - 2011, Pts 1 and 2, 1426 (2012) 4

[18] H.J. Qi, M.C. Boyce, Mech. Mater. 37, 817 (2005) 